TO APPEAR IN The Astrophysical Journal.

Preprint typeset using LATEX style emulateapj v. 26/01/00

\title{
THE CO TULLY-FISHER RELATION AND IMPLICATIONS FOR THE HOST GALAXIES OF HIGH-REDSHIFT QUASARS
}

\author{
LUIS C. Ho \\ The Observatories of the Carnegie Institution of Washington, 813 Santa Barbara St., Pasadena, CA 91101
}

To appear in The Astrophysical Journal.

\begin{abstract}
The integrated line width derived from CO spectroscopy provides a powerful tool to study the internal kinematics of extragalactic objects, including quasars at high redshift, provided that the observed line width can be properly translated to more conventionally used kinematical parameters of galaxies. We show, through construction of a $K_{s}$-band CO Tully-Fisher relation for nearby galaxies spanning a wide range in infrared luminosity, that the CO line width measured at $20 \%$ of the peak intensity, when corrected for inclination and other effects, successfully recovers the maximum rotation velocity of the disk. The line width at $50 \%$ of the peak intensity performs much more poorly, in large part because $\mathrm{CO}$ lines have a wide range of profiles, which are shown to vary systematically with infrared luminosity. We present a practical prescription for converting observed CO line widths into the stellar velocity dispersion of the bulge $\left(\sigma_{*}\right)$, and then apply it to a sample of low-redshift $(z \lesssim$ $0.2)$ and high-redshift $(1.4 \lesssim z \lesssim 6.4)$ quasars to study their host galaxies. Nearby quasars roughly fall on the correlation between black hole mass and bulge stellar velocity dispersion established for inactive galaxies, but the host galaxies of the high- $z$ quasars systematically deviate from the local $M_{\bullet}-\sigma_{*}$ relation. At a given $\sigma_{*}$, high- $z$ quasars have black hole masses larger by a factor of $\sim 4$ relative to local galaxies, suggesting that early in the life-cycle of galaxies the development of the bulge lags behind the growth of the central black hole. An alternative explanation for these observations, which currently cannot be ruled out rigorously, is that high-redshift quasars are preferentially viewed at face-on orientations.
\end{abstract}

Subject headings: galaxies: bulges — galaxies: ISM — galaxies: kinematics and dynamics — galaxies: nuclei — (galaxies:) quasars: general — galaxies: Seyfert

\section{INTRODUCTION}

The discovery of strong empirical relations between black hole $(\mathrm{BH})$ mass and the properties of nearby galaxies, both inactive (Kormendy \& Richstone 1995; Magorrian et al. 1998; Gebhardt et al. 2000a; Ferrarese \& Merritt 2000) and active (Gebhardt et al. 2000b; Ferrarese et al. 2001; Barth et al. 2005; Greene \& Ho 2006), has triggered an upsurge of interest in the coevolution of BHs and galaxies (e.g., Ho 2004). An important unanswered question is when the local scaling relations were established. While there is no shortage of opinions from theoretical studies (e.g., Croton 2006; Fontanot et al. 2006; Hopkins et al. 2007), to date solid empirical evidence has been relatively meager, and somewhat conflicting. From stellar velocity dispersion measurements of moderateluminosity active galactic nuclei (AGNs), Treu et al. (2004, 2007) and Woo et al. (2006) find that the $M_{\bullet}-\sigma_{*}$ relation at $z=0.36$ already begins to show departure from the local relation. On the other hand, studies of larger and more luminous AGN samples, but using less direct surrogates of $\sigma_{*}$ based on the widths of optical nebular emission lines, find little or no evolution of the $M_{\bullet}-\sigma_{*}$ relation out to $z \approx 1.2$ (Salviander et al. 2007) and $z \approx 3$ (Shields et al. 2003). At even higher redshifts, Walter et al. (2004) resolved the molecular gas in the $z=6.42$ quasar SDSS J114816.64+525150.3 into an apparent nuclear disk, from which they were able to derive a dynamical mass that, taken at face value, indicates that the host galaxy has an embryonic bulge compared to the estimated mass of the BH. Weiß et al. (2007) arrived at a very similar conclusion from their detailed study of the $z=3.91$ lensed quasar APM 08279+5255. While the interpretation of these results is not unambiguous, it qualitatively agrees with the photometric and lensing studies of high- $z$ quasars by Peng et al. (2006a, $2006 \mathrm{~b}$ ), which show that at $1 \lesssim z \lesssim 4.5$ the ratio of $\mathrm{BH}$ mass to host galaxy mass is a factor of several higher than the value in the local Universe.

The masses of high- $z$ quasar host galaxies are challenging to estimate accurately, and likely will remain so for some time. To make progress one should explore multiple avenues. One promising technique is to exploit the kinematic information encoded in molecular line observations. While studying molecular gas in high- $z$ sources is still far from routine, an increasing number of detections have been reported (Solomon \& Vanden Bout 2005, and references therein), and the upcoming generation of new millimeter-wave facilities hold great promise for rapid progress in this area. Shields et al. (2006) recently attempted to constrain the host galaxy masses of nine high- $z$ quasars with available measurements of the $\mathrm{CO}$ line width and $\mathrm{BH}$ mass. In order to place the high- $z$ objects on the $M_{\bullet}-\sigma_{*}$ relation, they calibrated the width of the CO line profile at $50 \%$ of the peak intensity (hereafter $W_{50}$ ) against a small sample of low- $z$ quasars for which they could obtain indirect estimates of the bulge stellar velocity dispersion, concluding in the end that $\sigma_{*}$ could be estimated simply from $W_{50} / 2.35$. Since the currently available high- $z$ quasars have conspicuously narrow $\mathrm{CO}$ lines (Greve et al. 2005; Carilli \& Wang 2006), Shields et al. (2006) suggest that these objects deviate strongly from the local $M_{\bullet}-\sigma_{*}$ relation in having a much larger $\mathrm{BH}$ mass for a given $\sigma_{*}$. The implication is that the growth of the $\mathrm{BH}$ predates, and is largely decoupled from, the formation of the galaxy bulge.

Although the interpretation of Shields et al. (2006) resonates well with the findings of Peng et al. (2006a, 2006b), it is not without complications. With a few exceptions (e.g., SDSS J114816.64+525150.3; Walter et al. 2004), most of 
High- $z$ Quasar Host Galaxies

the other quasars are not spatially resolved, and thus we have no knowledge of the spatial distribution of the molecular gas. Apart from signifying a shallow gravitational potential, a narrow $\mathrm{CO}$ line can arise from at least two other effects. First, the $\mathrm{CO}$ may be sufficiently concentrated toward the center of the host that it only samples the innermost part of the galaxy's rotation curve, thereby underestimating the maximum rotation velocity. This is well-known from observations (e.g., Downes \& Solomon 1998) as well as numerical simulations (e.g., Barnes \& Hernquist 1996) of merging galaxies, wherein the bulk of the molecular gas, as a result of tidal torques, sinks to the central few hundred parsecs and forms a compact nuclear disk or ring. As the hosts of high- $z$ quasars likely involve mergers, this should be a source of concern. Second, as argued by Carilli \& Wang (2006), the distribution of inclination angles for the CO-emitting disk of high- $z$ quasars, by virtue of their luminosity bias and selection method, may preferentially favor more face-on orientations. If so, then the inclination correction to the $\mathrm{CO}$ line widths can be arbitrarily large. For the high- $z$ sample to conform to the local $M_{\bullet}-\sigma_{*}$ relation, the average inclination angle needs to be $\langle i\rangle \approx 13-15^{\circ}$ (Carilli \& Wang 2006; Wu 2007). Lastly, we note that the interpretation of these and future observations depends quite critically on how one actually translates the $\mathrm{CO}$ line width into a more familiar dynamical variable. Shields et al. (2006) chose to calibrate $W_{50}$ with three indirect estimators of $\sigma_{*}$ (virial BH masses and [O III] $\lambda 5007$ line widths for seven objects, and host galaxy luminosities for two objects). By contrast, Wu (2007) examined directly the correlation between $W_{50}$ and $\sigma_{*}$ for a significantly larger sample of 33 Seyfert galaxies, concluding that the Shields et al. calibration is significantly nonlinear.

This paper presents a new method of estimating $\sigma_{*}$ based on more complete information on the $\mathrm{CO}$ line profile. We begin by asking three basic questions that we believe have not been adequately addressed. (1) How well does the CO line width trace the kinematics of galaxies and precisely what part of the galaxy does it probe? (2) What part of the CO profile, which, unlike that of H I is often not sharp and double-horned, should one measure? And (3) how do we relate the CO line width to the kinematics of the bulge? We investigate the first two issues by reexamining the CO Tully-Fisher relation, with particular emphasis on infrared-luminous galaxies, perhaps the best local analogs of what high- $z$ quasar host galaxies might look like. We find that galaxies over a wide range of infrared (IR) luminosities fall on the CO Tully-Fisher relation provided that one uses $W_{20}$, the width of the CO line at $20 \%$ of the peak intensity, rather than $W_{50}$. Moreover, we show that the shape of the line profile, as parameterized by the ratio $W_{20} / W_{50}$, varies significantly and systematically with IR luminosity. This thus provides a simple prescription for converting $W_{50}$ to the preferred parameter $W_{20}$ if the IR luminosity is known. Having shown that the CO line width can effectively probe the maximum rotation velocity $\left(v_{m}\right)$ of the galaxy, we then use the new calibration of the $v_{m}-\sigma_{*}$ relation by Ho (2007) and discuss the implications for high- $z$ quasar host galaxies.

\section{THE CO TULLY-FISHER RELATION FOR}

\section{INFRARED-LUMINOUS GALAXIES}

The CO Tully-Fisher relation is analogous to the more familiar relation commonly used with H I (Tully \& Fisher 1977). Originally proposed by Dickey \& Kasés (1992) and Sofue (1992), it has been applied to normal galaxies both nearby (Schöniger \& Sofue 1994, 1997; Lavezzi \& Dickey 1998) and at moderate distances up to $z \approx 0.1$ (Sofue et al. 1996; Tutui et al. 2001). As discussed by these authors, $\mathrm{CO}$ offers a number of potential advantages compared to $\mathrm{H} \mathrm{I}$, chief among them being the relative insensitivity of the $\mathrm{CO}$ distribution to environment perturbations and the technical feasibility of observing to greater distances.

One of the practical difficulties of using the CO line stems from the wide variety of line profiles actually observed. Unlike $\mathrm{HI}$, which generally exhibits a sharp-edged, double-horned profile characteristic of an optically thin rotating disk, $\mathrm{CO}$ line profiles are much more irregular, with shapes ranging from classic double peaks, to more rounded single peaks, to near Gaussians (e.g., Sanders et al. 1991; Dickey \& Kasés 1992). As discussed in Lavezzi \& Dickey (1997), many factors contribute to this diversity in $\mathrm{CO}$ line profiles, two physically interesting ones being the radial distribution of the gas and its opacity. In spiral galaxies the molecular gas is generally more centrally concentrated than the atomic hydrogen, and the ${ }^{12} \mathrm{CO}(1-0)$ transition tends to be optically thick. Nevertheless, in practice the full-width near zero intensity (or approximately $W_{20}$ ) of the CO line quite faithfully follows that of H I (Dickey \& Kasés 1992; Schöniger \& Sofue 1994). The molecular gas distribution, although more concentrated than the H I, evidently extends sufficiently past the peak of the rotation curve to imprint the maximum rotation velocity on the $\mathrm{CO}$ line profile. To achieve the best possible match between $\mathrm{CO}$ and $\mathrm{HI}$ velocities, one should apply corrections for interstellar turbulence (Lavezzi \& Dickey 1997), as well as accounting for the relative differences between the radial distribution of the two tracers and luminositydependent differences in the gradient of the rotation curve (Tutui \& Sofue 1999).

Do quasar host galaxies also obey the CO Tully-Fisher relation? Insofar as galaxy interactions or mergers are often implicated as the triggering mechanism of quasars (e.g., Hopkins et al. 2006), which are accompanied or preceded by starburst activity (e.g., Ho 2005), this issue can be effectively addressed by looking into the properties of nearby IR-luminous galaxies (Sanders \& Mirabel 1996). We have assembled a large sample of galaxies spanning a wide range in IR luminositie: 1 that contain measurements of both $W_{20}$ and $W_{50}$ for the ${ }^{12} \mathrm{CO}(1-$ 0 ) line integrated over at least a central diameter of $\sim 10 \mathrm{kpc}$, but preferably covering the entire galaxy. This last criterion is very important because most nearby galaxies are larger than the beam sizes of millimeter-wave telescopes, whereas we wish to simulate, as closely as possible, the global measurements typically achieved with single-dish H I telescopes in order to capture the full extent of the rotation curve. The rotation curves of spiral galaxies usually reach their maximum velocity at $r \approx 2-7$ $\mathrm{kpc}$, beyond which they remain flat (Sofue \& Rubin 2001). To achieve this, most of the objects are relatively distant $(60 \%$ are beyond $70 \mathrm{Mpc}$ ); more nearby systems have been mapped, and the published line widths represent the synthesized profile over the mapped positions. The final sample of 151 galaxies, assem-

\footnotetext{
${ }^{1}$ Throughout this paper $L_{\mathrm{IR}}$ refers to the total IR $(8-1000 \mu \mathrm{m})$ luminosity, derived using 12,25 , 60, and $100 \mu \mathrm{m}$ flux densities from the Infrared Astronomical Satellite (IRAS) following the prescription of Sanders \& Mirabel (1996). The IRAS data are taken from the NASA/IPAC Extragalactic Database (NED; http://nedwww.ipac.caltech.edu).
} 
TABLE 1: The Galaxy Sample

\begin{tabular}{|c|c|c|c|c|c|c|c|c|}
\hline $\begin{array}{c}\text { Galaxy } \\
\text { (1) }\end{array}$ & $\begin{array}{c}D_{L} \\
(\mathrm{Mpc}) \\
(2)\end{array}$ & $\begin{array}{l}i \\
\left({ }^{\circ}\right) \\
(3)\end{array}$ & $\begin{array}{c}W_{20}^{c} \\
\left(\mathrm{~km} \mathrm{~s}^{-1}\right) \\
(4)\end{array}$ & $\begin{array}{c}W_{50}^{c} \\
\left(\mathrm{~km} \mathrm{~s}^{-1}\right) \\
(5)\end{array}$ & $\begin{array}{c}M_{K_{s}} \\
(\mathrm{mag}) \\
(6)\end{array}$ & $\begin{array}{c}\log L_{\mathrm{IR}} \\
\left(L_{\odot}\right) \\
(7)\end{array}$ & $\begin{array}{c}\text { Reference } \\
\text { (8) }\end{array}$ & $\begin{array}{c}\text { Notes } \\
(9)\end{array}$ \\
\hline Arp 55 & 170.6 & 59: & 305 & 246 & $\ldots$ & 11.74 & 1 & \\
\hline Arp 148 & 150.7 & 40: & 551 & 342 & -23.09 & 11.63 & 1 & $\mathrm{~g}$ \\
\hline Arp 193 & 103.0 & 46: & 560 & 345 & -24.14 & 11.64 & 1 & \\
\hline Arp 220 & 80.1 & $\ldots$ & 1024 & 824 & -24.67 & 12.19 & 3 & \\
\hline CGCG 049-057 & 57.8 & 61 & 266 & 242 & -22.68 & 11.31 & 1 & \\
\hline
\end{tabular}

Note.- Col. (1) Galaxy name. Col. (2) Luminosity distance, derived from the mean heliocentric velocity listed in HyperLeda (http://leda. univ-lyon1.fr) assuming a Local Group infall velocity of $208 \mathrm{~km} \mathrm{~s}^{-1}$ toward the Virgo cluster and a Hubble constant of $H_{0}=70 \mathrm{~km} \mathrm{~s}^{-1} \mathrm{Mpc}^{-1}$. Col. (3) Inclination angle. Entries followed by a colon are especially uncertain because the galaxy morphology is severely disturbed. The inclination angles for the objects from Lavezzi \& Dickey 1998 are not listed, but they were applied by these authors to the line widths in Cols. (4) and (5). Col. (4) CO line width at $20 \%$ of the peak intensity, corrected for inclination angle and interstellar turbulence following the prescription of Lavazzi \& Dickey 1997. Note that these values of $W_{20}^{c}$ have not yet been corrected for the velocity-dependent effect discussed by Tutui \& Sofue (1999; see Eq. 1). Col. (5) Same as for Col. (4), but for the CO line width at $50 \%$ of the peak intensity. Col. (6) Total absolute $K_{s}$-band magnitude from 2MASS; these values have not been corrected for Galactic or internal extinction. Col. (7) Total IR $(8-1000 \mu \mathrm{m})$ luminosity. Col. (8) References for the CO data: (1) Sanders et al. 1991; (2) Schöniger \& Sofue 1994; (3) Lavazzi \& Dickey 1998. Col. (9) Notes: (a) Extreme low-velocity outlier in the Tully-Fisher relation; (b) IRAS $25 \mu \mathrm{m}$ upper limit; (c) IRAS $12 \mu \mathrm{m}$ upper limit; (d) K-band data from Kim et al. 2002; (e) Inclination angle from McLeod \& Rieke 1995; (f) Inclination angle from Scharwächter et al. 2003; (g) $K$-band data from Bushouse \& Stanford 1992; (h) $K$-band data from McLeod \& Rieke 1995; (i) $K$-band data from Bushouse \& Werner 1990. Table 1 is presented in its entirety in the electronic edition of the Astrophysical Journal. A portion is shown here for guidance regarding its form and content.

bled in Table 1 , covers $L_{\mathrm{IR}} \approx 10^{8}$ to $4 \times 10^{12} L_{\odot}$, ranging from nearby normal galaxies to those of the "luminous IR" and "ultraluminous IR" variety. The data were taken from three compilations: (1) luminous IRAS galaxies from Sanders et al. (1991); (2) nearby galaxies from Schöniger \& Sofue (1994), which in addition contains the two powerful AGNs Mrk 231 and I Zw 1 ; and (3) the distant cluster members and ultraluminous IR galaxies from Lavezzi \& Dickey (1998).

Inclination angles were estimated from axial ratios obtained mostly from de Vaucouleurs et al. (1991), and otherwise from Paturel et al. (2000), as described in Ho (2007), except that the intrinsic thickness of the disk was fixed at a value of 0.2 instead of allowing it to vary with Hubble type. The morphologies of many of the IR-luminous galaxies from the sample of Sanders et al. (1991) are particularly difficult to define, as a number of them are merger remnants or otherwise appear tidally disturbed. In Table 1 we have flagged the most suspicious values with a colon. To avoid excessively large and uncertain inclination corrections, we excluded objects with inclination angles less than $30^{\circ}$, except in the case of the Sanders et al. (1991) sample on account of the inherent difficulty of obtaining accurate inclinations for many of these sources. Following Lavezzi \& Dickey (1997), we apply a correction for turbulence broadening to the CO line widths, adopting their formalism and their recommended value of $38 \mathrm{~km} \mathrm{~s}^{-1}$ and $19 \mathrm{~km} \mathrm{~s}^{-1}$ for the correction to $W_{20}$ and $W_{50}$, respectively. [We did not apply corrections for inclination or turbulence broadening to the Lavezzi \& Dickey (1998) sample because these authors had already done so.] Lastly, we also apply the velocity-dependent correction recommended by Tutui \& Sofue (1999) to make $W_{20}$ fully compatible with the H I-based velocities. To account for the differ- ent radial distribution of $\mathrm{CO}$ versus $\mathrm{H} \mathrm{I}$ and for variations in the gradient of the rotation curve with luminosity (and hence rotation velocity), these authors suggest the following calibration:

$$
W_{20}^{c}=0.76 W_{20}+83.8
$$

We confirm that applying this additional correction does result in marked improvement in the final CO Tully-Fisher relation.

Figure 1 shows the $K_{s}$-band Tully-Fisher relation for the sample, plotting separately the maximum rotation velocity as estimated from the corrected $\mathrm{CO}$ line widths measured at $50 \%$ $\left(W_{50}^{c}\right)$ and $20 \%\left(W_{20}^{c}\right)$ of the line peak, and dividing the sample into normal and IR-luminous galaxies. With just a few exceptions, the $K_{s}$-band $(2.16 \mu \mathrm{m})$ magnitudes come from homogeneous photometry from the Extended Source Catalog of the Two-Micron All-Sky Survey (2MASS; Skrutskie et al. 2006). Overplotted on the figure is the H I Tully-Fisher relation derived by Verheijen (2001) for galaxies in the Ursa Major cluster in the $K^{\prime}$ band, which is quite similar to the 2MASS $K_{s}$ band (Bessel 2005). Looking first at the normal galaxies (top two panels), it is clear that $W_{20}^{c}$ fares better than $W_{50}^{c}$ in matching Verheijen's relation. Not only does the zeropoint agree better, the scatter is also reduced, from a root-mean-square (rms) of $1.0 \mathrm{mag}$ to $0.77 \mathrm{mag}$. A large part of the improvement stems from the fact that the Tutui-Sofue correction has been applied to $W_{20}$ but not to $W_{50}$ (because it is not currently known for the latter); without this correction, the scatter reduces only to an rms of $0.93 \mathrm{mag}$. The observed scatter for our sample is larger than Verheijen's, but this is hardly surprising considering the larger distance errors and greater heterogeneity of our sample. The bulk of the objects lie within the boundaries that enclose twice the rms 

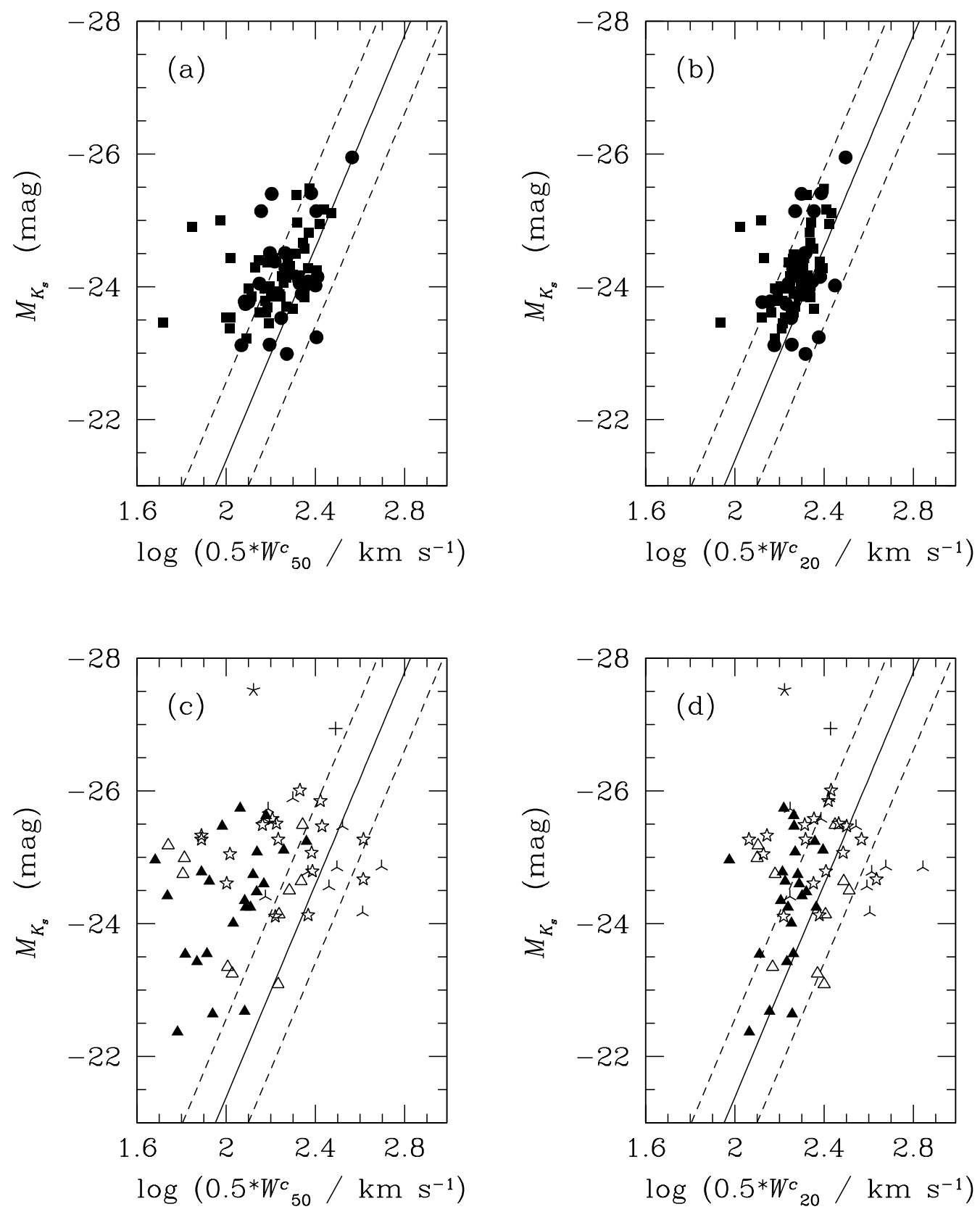

FIG. 1. - The CO Tully-Fisher relation in the $K_{s}$ band for (top) normal galaxies and (bottom) IR-luminous and IR-ultraluminous galaxies. The maximum rotation velocity is estimated using (left) $\frac{1}{2} W_{50}^{c}$ and (right) $\frac{1}{2} W_{20}^{c}$. The values of $W_{50}^{c}$ and $W_{20}^{c}$ have been corrected for inclination and turbulence, and $W_{20}^{c}$ has been corrected in addition for the Tutui-Sofue (1999) effect (see Eq. 1). In the top panels the circles are the nearby galaxies from Schöniger \& Sofue (1994), and the squares are the cluster galaxies from Lavezzi \& Dickey (1998); only objects with $i \geq 30^{\circ}$ are included. In the bottom panels, the triangles come from the sample of Sanders et al. (1991), where the solid symbols represent objects without obvious optical morphological peculiarities, open symbols represent those with clearly disturbed morphologies or tidal features, and skeletal symbols denote those with inclination angles formally less than $30^{\circ}$. The open stars mark the 21 ultraluminous IR galaxies from the study of Solomon et al. (1997) for which Lavezzi \& Dickey (1998) obtained follow-up optical imaging to determine inclination angles. Mrk 231 and I Zw 1 from Schöniger \& Sofue (1994) are plotted as an asterisk and a plus symbol, respectively. The solid line shows the $K^{\prime}$-band Tully-Fisher relation from Verheijen (2001) based on H I rotation curves of spiral galaxies in the Ursa Major cluster; the dashed lines lines mark the region that has twice the rms scatter of the Ursa Major objects.

scatter of Verheijen's "H I" sample (0.59 mag). There appears to be a small zeropoint offset of $\sim 0.3 \mathrm{mag}$ (or $\sim 9 \%$ increase in velocity), but given the limited size and character of our sample, we regard this finding as tentative. Overall, our analysis reaffirms the results of previous attempts to compare the $\mathrm{CO}$ and $\mathrm{H}$ I Tully-Fisher relations at optical wavelengths, now extended for the first time to the near-IR.

That $W_{20}^{c}$ is far superior than $W_{50}^{c}$ in recovering the maximum rotation velocity is most dramatically illustrated for the sample of IR-luminous galaxies shown on the bottom two panels of Figure 1. Employing $W_{50}^{c}$ places the bulk of the objects significantly offset (by $\sim 2 \mathrm{mag}$ ), with enormous scatter ( $\mathrm{rms}=1.9$ 
mag), to the low-velocity side of the reference Tully-Fisher locus. The use of $W_{20}^{c}$, on the other hand, shifts most of the objects back to the fiducial ridgeline and markedly tightens the distribution (rms $=1.5 \mathrm{mag}$ ). Again, the Tutui-Sofue correction to $W_{20}$ has a significant impact; had it not been applied, the rms scatter is reduced only to $1.7 \mathrm{mag}$. To be sure, the scatter is larger than in the case of the normal galaxies, but this is entirely to be expected given how poorly we know the inclination angles, not to mention of the strong tidal perturbations witnessed in the stars, which presumably must also be imprinted to some degree on the gas. There are no clear-cut differences when the sample is separated either by the degree of morphological peculiarity as judged by our qualitative visual examination of optical images in NED (open versus solid triangles) or by the more detailed "interaction types" given in Sanders et al. (1991), nor do the highest luminosity sources (open stars) stand out in any conspicuous manner. Despite the appreciable star formation rates in many of these systems, most of which qualify as hefty starbursts, we see no luminosity offset relative to the fiducial ridgeline. The modest amount of brightening predicted in the $K$ band for starbursts ( $\sim 0.5$ mag; Mouhcine \& Lançon 2003) may be imperceptible in the midst of the large scatter in the figure.

As an aside, we note that our sample of normal galaxies (Fig. 1b) contains 3-4 outliers with exceptionally low rotation velocities, reminiscent of the population of kinematically anomalous galaxies identified by Ho (2007) on the basis of H I measurements. Ho posits that these objects possess substantial amounts of dynamically unrelaxed atomic hydrogen captured either from minor mergers or possibly accreted directly from primordial clouds. However, in view of the substantial quantity of molecular gas involved in the current sample, a primordial origin seems far-fetched. Instead, we may appeal to the other arguments presented by Ho (2007), namely that the gas and stars might be strongly misaligned, or else the gas has been severely perturbed by tidal interactions. Since all of the outliers belong to the cluster sample of Lavezzi \& Dickey (1998), this is perhaps not implausible. The IR-luminous subsample (Fig. 1d) also contains a number of low-velocity outliers, but these are balanced by an almost equal number of high-velocity outliers, a situation that can be explained by the gas and stars not being coplanar (Ho 2007). Given the great difficulty in estimating inclination angles for this subsample, however, we should not attach too much weight to the observed scatter.

In the context of this study, the critical point of the above analysis is that the $\mathrm{CO}$ line width, as measured by $W_{20}^{c}$, provides a robust measurement of the maximum rotation velocity of the large-scale disk of the galaxy, on nearly equal footing with velocities more conventionally derived from integrated $\mathrm{H} \mathrm{I}$ line widths or extended $\mathrm{H} \mathrm{I}$ or $\mathrm{H} \alpha$ rotation curves. This holds not only for normal galaxies, but also for IR-luminous systems, whose evolutionary status may be hoped to bear some resemblance to that of quasar host galaxies.

\section{ESTIMATING $\sigma_{*}$ FROM CO LINE WIDTHS}

We have established that the width of the CO line measured at $20 \%$ of the peak intensity, $W_{20}^{c}$, accurately recovers the maximum rotation velocity of the disk, $v_{m}$. On the other hand, the kinematic quantity most closely linked to BH mass is the velocity dispersion of the bulge, $\sigma_{*}$, not $v_{m}$. A number of studies (e.g., Ferrarese 2002; Pizzella et al. 2005) have suggested that galaxies over a wide range of morphological types obey a tight, nearly linear correlation between $v_{m}$ and $\sigma_{*}$, and by virtue of the $M_{\bullet}-\sigma_{*}$ relation, that $\mathrm{BH}$ mass may be equally, or perhaps even more fundamentally, coupled to $v_{m}$ than to $\sigma_{*}$. The recent analysis of Ho (2007; see also Courteau et al. 2007), however, shows that in fact the $v_{m}-\sigma_{*}$ relation exhibits substantial intrinsic scatter and that its zeropoint varies systematically with galaxy morphology, bulge-to-disk ratio, and light concentration. Despite these complications, $v_{m}$ is correlated with $\sigma_{*}$, both as observed, not only for inactive but also active galaxies (Ho et al. 2007), and as anticipated from basic dynamical considerations (see discussion in Ho 2007), and so we can take advantage of this fact to translate the $\mathrm{CO}$ line width into $\sigma_{*}$. Ho (2007) presented fits to the $v_{m}-\sigma_{0}$ relation using the largest and most comprehensive calibration sample to date. Since the zeropoint depends on morphological type, and the scatter is somewhat reduced for later-type galaxies, we use here the fit for the 550 "kinematically normal" spiral galaxies in Ho's sample (Eq. 5 in Ho 2007), to yield

$$
\log \sigma_{*}=(1.26 \pm 0.046) \log v_{m}-(0.78 \pm 0.11),
$$

where in the present context $v_{m} \approx \frac{1}{2} W_{20}^{c}$.

Despite the advantages of $W_{20}^{c}$ over $W_{50}^{c}$, two practical difficulties often preclude using the former over the latter. The line width near the base of the profile is maximally sensitive to the noise in the spectrum and to errors in baseline determination, and hence $W_{20}$ is inherently more uncertain than $W_{50}$. Moreover, many studies, presumably for this very reason, do not even publish $W_{20}$, but $W_{50}$ is often given. Now, $W_{20}$ can be estimated from $W_{50}$ in two simple cases: for a sharp-edged, double-horned profile $W_{20} / W_{50} \approx 1$, and for a Gaussian $W_{20} / W_{50} \approx 1.5$. Many CO profiles, however, do not conform to these two simple shapes, and indeed real line profiles can even have wings more extended than a Gaussian, and so it would be highly desirable to devise an empirical guideline to estimate $W_{20}$ from $W_{50}$. Note that the differences between these two examples have dramatic consequences for the inferred $\mathrm{BH}$ masses. A 50\% increase in the $\mathrm{CO}$ line width, for example, when folded through Equation 2 and the $M_{\bullet}-\sigma_{*}$ relation of Tremaine et al. $\left(2002 ; M_{\bullet} \propto \sigma_{*}^{4.02}\right)$, results in a factor of $\sim 8$ increase in the predicted $\mathrm{BH}$ mass.

Recall from our discussion of Figure 1 that the impact of substituting $W_{50}^{c}$ with $W_{20}^{c}$ had a much more dramatic effect in recovering the H I Tully-Fisher relation in the case of the IRluminous galaxies than for normal galaxies. The implication is that the line shape must vary systematically with IR luminosity. We illustrate this effect in Figure 2, where we plot $W_{20}^{c} / W_{50}^{c}$ as a function of the total IR luminosity. At $L_{\mathrm{IR}} \lesssim 10^{10} L_{\odot}$, $W_{20}^{c} / W_{50}^{c} \approx 1$, but $W_{20}^{c} / W_{50}^{c}$ progressively increases toward higher luminosities, such that by $L_{\mathrm{IR}} \approx 10^{12} L_{\odot}$ it attains a median value close to 1.5 , the expected value for a Gaussian adopted by Shields et al. (2006). Despite the large scatter and the fact that $W_{20}^{c} / W_{50}^{c}$ is bounded on one side, a generalized Spearman rank-order correlation test formally yields a correlation coefficient of $\rho=0.48$, with a probability of $<10^{-4}$ for rejecting the null hypothesis of no correlation. A linear regression fit with $\log L_{\mathrm{IR}}$ as the independent variable gives

$$
W_{20}^{c} / W_{50}^{c}=(0.21 \pm 0.041) \log L_{\mathrm{IR}}-(1.05 \pm 0.44) .
$$

Note that at any given luminosity there are objects spanning the full range of $W_{20}^{c} / W_{50}^{c}$, from $\sim 1$ up to a maximum value that increases roughly monotonically with luminosity. It is the upper envelope of the $W_{20}^{c} / W_{50}^{c}$ distribution, and consequently the 


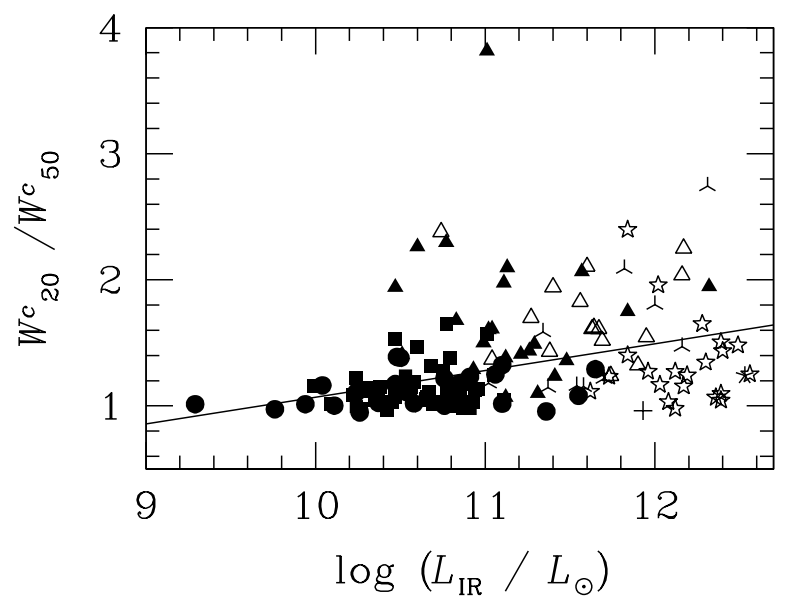

FIG. 2.- Variation of the line shape, as parameterized by the ratio $W_{20}^{c} / W_{50}^{c}$, as a function of total IR luminosity. The symbols have the same meaning as in Figure 1. The solid line shows the linear regression fit between $W_{20}^{c} / W_{50}^{c}$ and $\log L_{\mathrm{IR}}$ (Eq. 3)

dispersion about the mean, that increases with luminosity; at the highest luminosities $W_{20}^{c} / W_{50}^{c}$ can be as large as 2-2.5. Since physically $W_{20}^{c}$ should be larger than $W_{50}^{c}$, even if measurement errors may result in $W_{20}^{c} / W_{50}^{c} \lesssim 1$, Equation 3 strictly speaking should only be applied when $W_{20}^{c} / W_{50}^{c}>1$. Also, it is worth stressing that the correction recommended in Equation 3 is statistical in nature; given the large scatter about the mean relation, especially at high luminosities, applying the correction to any individual source is subject to a large uncertainty.

Interestingly, Lavezzi \& Dickey (1997) searched for possible correlations of $\mathrm{CO}$ line shape with galaxy properties but found none that was especially compelling. It could be that the effect described here was masked by their smaller sample and more limited dynamic range. Multiple factors influence the observed CO line profile (Dickey \& Kasés 1992; Lavezzi \& Dickey 1997), but chief among them are the optical depth and the radial distribution of the gas. The optical depth of the ${ }^{12} \mathrm{CO}(1-0)$ line in starbursts, however, if anything, appears to be lower than in normal galaxies (e.g., Aalto et al. 1995; Glenn \& Hunter 2001), in which case Figure 2 actually exhibits the opposite trend predicted if opacity were the principal cause for the line shape variation. On the other hand, it is entirely natural to expect the molecular gas to be more centrally concentrated in starburst galaxies. The most powerful starbursts seem to arise from central stockpiles of molecular gas that have been dissipated to the nuclear region, often with the aid of tidal interactions (e.g., Barnes \& Hernquist 1996; Sanders \& Mirabel 1996; Downes \& Solomon 1998).

In summary, we recommend the following recipe for converting an observed $\mathrm{CO}$ line width into $\sigma_{*}$. This recipe is empirically motivated by the success with which we were able to recover the rotational velocity of IR-luminous galaxies, likely good analogs of quasars, as described in Section 2. If the line width at $20 \%$ of the peak can be reliably measured, then $W_{20}$ should be used, after correcting it for turbulence as described in Lavezzi \& Dickey (1997), inclination, and the Tutui-Sofue (1999) effect (Eq. 1). If only the width at $50 \%$ of the peak is known or can be measured with confidence, then after correcting for turbulence and inclination, Equation 3 should be used in concert with an estimate of $L_{\mathrm{IR}}$ to convert $W_{50}^{c}$ to $W_{20}^{c}$, followed by an adjustment for the Tutui-Sofue (1999) effect. If the incli- nation angle of the source is unknown, as is almost always the case in high- $z$ objects, but can be assumed to be random, then $\langle i\rangle=45^{\circ}$. With $W_{20}^{c}$ at hand, $\sigma_{*}$ follows from the $v_{m}-\sigma_{*}$ relation (Eq. 2).

\section{THE HOST GALAXIES OF HIGH-Z QUASARS}

We apply the recipe outlined above to reassess the $M_{\bullet}-\sigma_{*}$ relation of high- $z$ quasars. For comparison, we also evaluate all the low- $z$ quasars with available $\mathrm{CO}$ line widths. The data, compiled in Table 2, are similar to those presented in Shields et al. (2006), but with several important modifications. First, we expanded the sample of low- $z$ Palomar-Green (PG) quasars from 12 to 17 . The five additional sources have uniformly measured broad $\mathrm{H} \beta$ line widths from Boroson \& Green (1992) and optical spectrophotometry from Neugebauer et al. (1987), and so BH masses can be derived for them using the conventional virial method. The line widths from the original sample of Evans et al. (2001) have now been updated by Evans et al. (2006). For ease of comparison, we adopt the particular virial relation used by Shields et al., as well as their choice of cosmological parameters to derive distances. One key difference lies in the treatment of the $\mathrm{CO}$ line widths. The line widths for the nine objects published in Scoville et al. (2003) pertain to the full-width near zero intensity $\left(\sim W_{20}\right)$, not the full-width at half maximum $\left(W_{50}\right)$. Shields et al. obtained the $W_{50}$ values of these sources by assuming that $W_{50}=\frac{2}{3} W_{20}$. Since the formalism developed in this paper explicitly accounts for line shape variations, Table 2 carefully documents the original data. We also found it necessary to adjust the line width data for a few of the high- $z$ quasars.

Figure 3 summarizes the $\mathrm{CO}$-derived $M_{\bullet}-\sigma_{*}$ relation for quasars, where we have applied a correction for an average inclination angle of $45^{\circ}$. The low $z$ PG quasars roughly center around the local $M_{\bullet}-\sigma_{*}$ relation of inactive galaxies (Tremaine et al. 2002), but the scatter is very large. Taken at face value, the PG quasars actually lie slightly below the local relation, reminiscent of a similar tendency reported in other broad-line AGNs (Greene \& Ho 2006). We refrain from quantifying the exact magnitude of the offset, as it depends on the choice of the virial relation used to obtain the $\mathrm{BH}$ mass, and because the current sample is both small and compromised by the lack of inclination angle information. Two of the objects have exceptionally narrow CO lines (PG $0838+770, W_{50}=60 \mathrm{~km} \mathrm{~s}^{-1}$; PG $1415+451, W_{50}=90 \mathrm{~km} \mathrm{~s}^{-1}$ ), but they should not be viewed as too alarming, since similar cases are seen among inactive normal and IR-luminous galaxies. Both of these quasars possess luminous hosts $\left(M_{K} \approx-25.8\right.$ and -24.7 mag, respectively, assuming $H-K \approx 0.3 \mathrm{mag}$; McLeod \& Rieke 1994), which would place them among the kinematically anomalous members in the CO Tully-Fisher relation (Fig. 1b).

More interesting is the situation for the high- $z$ quasars. Consistent with the analysis of Shields et al. (2006), high- $z$ quasars seem to show a clear statistical offset above the local $M_{\bullet}-\sigma_{*}$ relation. Our treatment of the $\mathrm{CO}$ line widths, however, has reduced considerably the magnitude of the offset, from an average value of $\Delta \log M_{\mathrm{BH}}=1.8 \mathrm{dex}$ as found by Shields et al. to $\Delta \log M_{\mathrm{BH}} \approx 0.6 \mathrm{dex}$. This brings these results into better agreement with the findings of Peng et al. (2006b), whose photometric analysis of high- $z$ quasar host galaxies suggests that at $1.7 \lesssim z \lesssim 4.5$ the ratio of $\mathrm{BH}$ mass to bulge stellar mass is a factor of 4-6 higher than the local value. Of course, without 
TABle 2: The Quasar SAmple

\begin{tabular}{|c|c|c|c|c|c|c|c|c|c|}
\hline $\begin{array}{c}\text { Quasar } \\
\text { (1) }\end{array}$ & $\begin{array}{l}z \\
(2)\end{array}$ & $\begin{array}{c}\log M_{\mathrm{BH}} \\
\left(M_{\odot}\right) \\
(3)\end{array}$ & $\begin{array}{c}W_{20} \\
\left(\mathrm{~km} \mathrm{~s}^{-1}\right) \\
(4)\end{array}$ & $\begin{array}{c}W_{50} \\
\left(\mathrm{~km} \mathrm{~s}^{-1}\right) \\
(5)\end{array}$ & $\begin{array}{c}W_{20}^{c} \\
\left(\mathrm{~km} \mathrm{~s}^{-1}\right) \\
(6)\end{array}$ & $\begin{array}{c}\sigma_{*} \\
\left(\mathrm{~km} \mathrm{~s}^{-1}\right) \\
(7)\end{array}$ & $\begin{array}{c}\log L_{\mathrm{IR}} \\
\left(L_{\odot}\right) \\
(8)\end{array}$ & $\begin{array}{c}\text { Reference } \\
\text { (9) }\end{array}$ & $\begin{array}{c}\text { Notes } \\
(10)\end{array}$ \\
\hline PG $0050+124$ & 0.0611 & 6.95 & 405 & 400 & 596 & 218 & 11.93 & 1 & $\mathrm{a}$ \\
\hline PG $0052+251$ & 0.171 & 8.61 & 500 & $\ldots$ & 580 & 210 & 11.72 & 3 & $\mathrm{~b}$ \\
\hline PG $0157+001$ & 0.163 & 8.02 & $\ldots$ & 270 & 517 & 182 & 12.65 & 4 & \\
\hline PG $0804+761$ & 0.100 & 8.02 & 881 & $\ldots$ & 990 & 412 & 11.72 & 2 & \\
\hline PG $0838+770$ & 0.131 & 7.71 & $\ldots$ & 60 & 164 & 43 & 11.60 & 4 & \\
\hline PG $1119+120$ & 0.0502 & 7.22 & $\ldots$ & 220 & 364 & 117 & 11.16 & 4 & \\
\hline PG $1229+204$ & 0.0630 & 7.87 & 266 & $\ldots$ & 329 & 103 & 11.26 & 2 & \\
\hline PG $1351+640$ & 0.088 & 8.67 & 414 & $\cdots$ & 488 & 169 & 11.87 & 2 & \\
\hline PG $1404+226$ & 0.0980 & 6.71 & 468 & $\ldots$ & 546 & 195 & 11.12 & 2 & \\
\hline PG $1415+451$ & 0.114 & 7.68 & $\ldots$ & 90 & 203 & 56 & 11.50 & 4 & \\
\hline PG $1426+015$ & 0.0865 & 8.63 & 633 & $\ldots$ & 723 & 278 & 11.51 & 2 & \\
\hline PG $1440+356$ & 0.0791 & 7.18 & 600 & $\cdots$ & 688 & 261 & 11.57 & 2 & \\
\hline PG $1444+407$ & 0.267 & 8.14 & 300 & $\cdots$ & 366 & 118 & 12.34 & 3 & $\mathrm{~b}$ \\
\hline PG $1613+658$ & 0.129 & 8.94 & $\cdots$ & 400 & 688 & 260 & 12.02 & 4 & \\
\hline PG 2130+099 & 0.0629 & 7.61 & 487 & $\cdots$ & 566 & 204 & 11.45 & 2 & \\
\hline PG 2214+139 & 0.0658 & 8.25 & 488 & $\cdots$ & 567 & 205 & 11.08 & 2 & \\
\hline Q $0957+561$ & 1.412 & 8.86 & 660 & $\cdots$ & 752 & 292 & 12.81 & 5 & c \\
\hline Cloverleaf & 2.558 & 8.2 & $\cdots$ & 416 & 777 & 304 & 12.73 & 6 & d \\
\hline RXJ J0911+0551 & 2.796 & 8.85 & $\cdots$ & 350 & 634 & 235 & 12.36 & 7 & \\
\hline SMM J04135+1027 & 2.846 & 8.82 & $\cdots$ & 340 & 691 & 262 & 13.38 & 7 & \\
\hline APM $08279+5255$ & 3.911 & 10.36 & $\cdots$ & 475 & 955 & 394 & 13.46 & 8 & $\mathrm{e}$ \\
\hline BRI 1335-0417 & 4.407 & 9.77 & $\ldots$ & 420 & 849 & 340 & 13.45 & 7 & \\
\hline BRI 0952-0115 & 4.434 & 9.27 & $\cdots$ & 230 & 436 & 147 & 12.38 & 7 & \\
\hline BRI 1202-0725 & 4.694 & 10.16 & $\cdots$ & 333 & 711 & 272 & 13.85 & 9 & $\mathrm{f}$ \\
\hline SDSS J1148+5251 & 6.419 & 9.37 & $\cdots$ & 279 & 579 & 210 & 13.43 & 10 & $\mathrm{~g}$ \\
\hline
\end{tabular}

Note.- Col. (1) Quasar name. Col. (2) Redshift. We adopted the luminosity distances used by Shields et al. 2006, which are based on $H_{0}=70 \mathrm{~km} \mathrm{~s}^{-1} \mathrm{Mpc}^{-1}, \Omega_{\mathrm{m}}=0.3$, and $\Omega_{\Lambda}=0.7$. Col. (3) BH mass taken from Shields et al. 2006, except for the following four cases (PG 0052+251, 1351+640, 1444+407, and 0157+001), whose BH masses were derived using Equation 2 from Shields et al., H $\beta$ FWHM from Boroson \& Green 1992, and $5100 \AA$ continuum luminosities from the spectrophotometry of Neugebauer et al. 1987. Col. (4) CO line width at $20 \%$ of the peak intensity. Col. (5) CO line width at $50 \%$ of the peak intensity. Col. (6) $W_{20}$ corrected for turbulence, an average inclination angle of $45^{\circ}$, the Tutui-Sofue effect, and, in the case of $W_{50}$, the luminosity dependence on line shape (see §3) using the IR luminosities from Col. (8). Col. (7) Stellar velocity dispersion of the bulge derived from $W_{20}^{c}$ and the $v_{m}-\sigma_{*}$ relation. Col. (8) Total IR (8-1000 $\left.\mu \mathrm{m}\right)$ luminosity. In the case of the high- $z$ quasars, they are approximated by the far-IR luminosities taken from Solomon \& Vanden Bout 2005; the luminosities of known lensed sources have been corrected for lensing magnification. Col. (9) Reference for the CO line width: (1) Schöniger \& Sofue 1994; (2) Scoville et al. 2003; (3) Casoli \& Loinard 2001; (4) Evans et al. 2006; (5) Planesas et al. 1999; (6) Weiß et al. 2003; (7) Solomon \& Vanden Bout 2005; (8) Weiß et al. 2007; (9) Reichers et al. 2006; (10) Bertoldi et al. 2003. Col. (10) Notes: (a) I Zw 1 from Table $1 ; W_{20}^{c}$ corrected for $i=38^{\circ}$. (b) $W_{20}$ measured manually from an enlarged version of the published plot; uncertainty $\sim 100 \mathrm{~km} \mathrm{~s}^{-1}$. (c) Solomon \& Vanden Bout 2005 quote $W_{50}=280 \mathrm{~km}$ $\mathrm{s}^{-1}$ from Krips et al. 2004, but no line width information is actually given in the latter. Greve et al. 2005 attribute $W_{50}=$ $440 \mathrm{~km} \mathrm{~s}^{-1}$ to Planesas et al. 1999 and Krips et al. 2004, but it is unclear how this value was deduced. The lensing model of Krips et al. suggests that the double-peaked profile of component "CO-A" arises from the disk of the host galaxy. We thus use the centroid velocities of the two peaks $\left(-160\right.$ and $\left.+500 \mathrm{~km} \mathrm{~s}^{-1}\right)$ as given in Planesas et al. 1999 to infer a full velocity width of $660 \mathrm{~km} \mathrm{~s}^{-1}$, which we approximate as $W_{20}$. (d) The $W_{50}$ value from Weiß et al. 2003 has the smallest error bar; our adopted value differs slightly from the value of $400 \mathrm{~km} \mathrm{~s}^{-1}$ used by Shields et al. 2006. (e) $W_{50}$ from the average value of $475 \pm 17 \mathrm{~km} \mathrm{~s}^{-1}$ obtained using the IRAM Plateau de Bure interferometer, which is better determined than the value from the IRAM $30 \mathrm{~m}$. (f) $W_{50}$ differs from the value of $350 \mathrm{~km} \mathrm{~s}^{-1}$ used by Shields et al. 2006. (g) Solomon \& Vanden Bout 2005 quote $W_{50}=250 \mathrm{~km} \mathrm{~s}^{-1}$ from Walter et al. 2003, who fit a Gaussian profile to the combined $\mathrm{CO}(6-5)$ and $\mathrm{CO}(7-6)$ spectra from Bertoldi et al. 2003. The published version of Bertoldi et al.'s paper, however, actually gives $W_{50}=279 \mathrm{~km} \mathrm{~s}-1$, which we adopt here.

actual observational constraints on inclination angles, it is impossible to disprove the prosaic alternative that these sources are simply biased toward more face-on orientations. To shift the ensemble of high- $z$ points back to the local $M_{\bullet}-\sigma_{*}$ relation would require the average inclination angle to be $\langle i\rangle=30^{\circ}$ 
instead of $45^{\circ}$, less extreme than $\langle i\rangle=15^{\circ}$ found by Wu (2007).

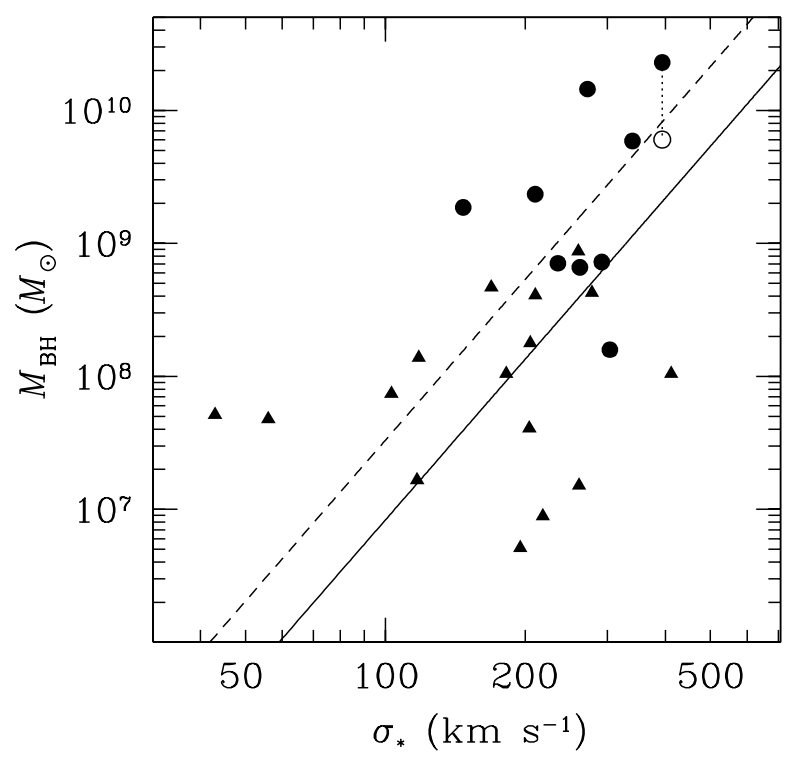

FIG. 3.- The $M_{\bullet}-\sigma_{*}$ relation for low- $z$ PG quasars (triangles) and high$z$ quasars (circles). The $\mathrm{BH}$ masses were derived from the quasar line width and luminosity using the virial technique, and velocity dispersion comes from the $\mathrm{CO}$ line width. The point marked with an open circle and connected with a dotted line shows the new location of APM 08279+5255 with its BH mass adjusted to the revised luminosity lensing magnification factor of Weiß et al. (2007). The solid line shows the $M_{\bullet}-\sigma_{*}$ relation of Tremaine et al. (2002) for local inactive galaxies, and the dashed line shows an offset of 0.6 dex in mass from the local relation.

Despite this impasse, the good agreement between the present kinematic analysis and the photometric study of Peng et al. (2006b) lends credence to the proposition that at high redshifts the growth of the $\mathrm{BH}$ outpaced the growth of the bulge.

We end with a few cautionary remarks, which we hope can serve as a catalyst for future work. First, we should recognize that BH masses derived from the virial method carry substantial uncertainty. This is especially so for masses that rely on the C IV $\lambda 1549$ line, which might be biased with respect to masses obtained from $\mathrm{H} \beta$ (Baskin \& Laor 2005). It would be highly desirable to acquire high-quality near-IR spectra of all CO-detected high- $z$ quasars in order to reevaluate their $\mathrm{BH}$ masses using an internally consistent, homogeneous set of line measurements based on either $\mathrm{H} \beta$ or Mg II $\lambda 2800$ (e.g., Barth et al. 2003). A number of the high- $z$ quasars are known or suspected to be lensed. More accurate lensing models would help to bolster our confidence in their intrinsic luminosities, which enter crucially into the $\mathrm{BH}$ mass estimate via the sizeluminosity relation. Two of the objects in Table 2 have uncomfortably large $\mathrm{BH}$ masses $\left(M_{\bullet}>10^{10} M_{\odot}\right)$, larger than any directly measured in the local Universe. BRI $1202-0725$ is not known to be lensed, but APM $08279+5255$ is. Weiß et al. (2007) recently revised the luminosity magnification factor of APM $08279+5255$ from 7, the value given in Solomon $\&$ Vanden Bout (2005) that Shields et al. (2006) adopted, to 100 . Since the virial mass scaling relation used by Shields et al. depends on $L^{0.5}$, reducing the luminosity by a factor of 14 lowers the BH mass by a factor $\sim 4$ (see Fig. 3). At the moment we do not know how many of the other lensed objects may be similarly affected.

The correction for line shape that we advocate in this study
(Eq. 3) depends on the total IR luminosity, but we have not specified what actually powers this luminosity. Many IRluminous galaxies are composite systems whose bolometric luminosity comes from a mixture of AGN heating and star formation. If the empirical line shape correction is driven largely by the starburst component to $L_{\mathrm{IR}}$, then we may have conceivably overcorrected the CO line widths for the quasars, whose IR luminosity is at least partly, and some would argue predominantly (Ho 2005), due to AGN heating. This issue can be addressed by obtaining quantitative estimates of the star formation rate in these systems, for instance using the technique proposed by Ho \& Keto (2007). A more straightforward remedy, of course, is to simply take better $\mathrm{CO}$ spectra so that $W_{20}$ can be directly measured.

Finally, the apparently excellent agreement between our results and those of Peng et al. (2006b) is somewhat misleading. High-redshift galaxies have steeper gravitational potential wells, and so a given $\sigma_{*}$ corresponds to a smaller bulge stellar mass (Robertson et al. 2006). The factor of $\sim 4$ increase in BH mass for high- $z$ quasars compared to the local $M_{\bullet}-\sigma_{*}$ relation, therefore, actually results in an ever larger offset had the comparison been done in terms of stellar mass, which is the approach taken by Peng et al. (2006b). On the other hand, Peng et al.'s analysis assumed a rather conservative mass-to-light ratio, one designed to yield the largest bulge mass. In this sense the factor of 4-6 increase in the BH-to-bulge mass ratio found by Peng et al. should be viewed as a lower limit (C. Y. Peng 2007, private communications), which brings their results in closer agreement with ours.

\section{DISCUSSION AND SUMMARY}

The increasing realization that BHs and their energy feedback play a fundamental role in the life-cycle of galaxies places great urgency in devising effective methods to diagnose high- $z$ quasars and their hosts. Many approaches can be taken to tackle this problem, but a particularly direct one is to attempt to detect evolution in the well-known local scaling relations between $\mathrm{BH}$ mass and host galaxy properties. The host galaxies of high$z$ quasars, however, pose a formidable challenge for studying in stellar light. Not only must one contend with the enormous glare of the luminous AGN, but one is further handicapped by surface brightness dimming, band-pass shifting, and the uncertain star formation history of the galaxy. While the photometric properties of the stars can be studied under optimal conditions (e.g., in lensed systems; Peng et al. 2006b), stellar kinematical information will be much more difficult to come by, even in the foreseeable future. Within this backdrop, observations of the large-scale cold interstellar medium of the host can serve as a powerful tool to probe not only its gas content but its global kinematics (Ho et al. 2007). From experience with galaxies in the nearby Universe, even a measurement as crude as an integrated spectrum can yield an important physical parameter, namely the rotation velocity of the disk. The recent progress in the detection of $\mathrm{CO}$ emission in high- $z$ quasars (Solomon $\&$ Vanden Bout 2005) presents an opportunity to explore this problem.

Since the $M_{\bullet}-\sigma_{*}$ relation serves as the best local reference benchmark, we must devise a method to relate CO line widths to $\sigma_{*}$. Shields et al. (2006) tried a number of proxies, but were unconvinced as to which was most reliable, in large 
part because their calibration samples were very small and because each of the empirical correlations they employed has significant intrinsic scatter. In the end they simply assumed $\sigma_{*}$ $\approx W_{50}(\mathrm{CO}) / 2.35$. Wu (2007), by contrast, directly compared $W_{50}(\mathrm{CO})$ with $\sigma_{*}$ measurements for 33 Seyfert galaxies to arrive at a conversion between the two.

We have investigated the $\mathrm{CO}$ line width calibration problem from a very different perspective. We began by showing that normal galaxies obey a tight CO Tully-Fisher relation in the $K_{s}$ band, and that we can recover the more familiar H I-based relation provided that we use the line width measured at $20 \%$ of the peak $\left(W_{20}\right)$ instead of that measured at $50 \%$ of the peak $\left(W_{50}\right)$. The reduction in the scatter is especially impressive for IRluminous galaxies, whose frequently disturbed morphologies, as a result of tidal interactions and mergers, produce a wide array of $\mathrm{CO}$ line shapes, and hence large differences between $W_{20}$ and $W_{50}$. Indeed, we show that the line shape, as gauged by $W_{20} / W_{50}$, varies substantially and systematically with IR luminosity. We further discuss additional corrections that need to be applied to the line widths in order to achieve the best possible results. Having established $W_{20}$ to be superior to $W_{50}$ as a measure of maximum rotation velocity $\left(v_{m}\right)$ of the disk, as well as an empirical guideline to convert $W_{50}$ to $W_{20}$ if the latter is not available, we then turn to the issue of how to relate $W_{20}$ (or equivalently $v_{m}$ ) to $\sigma_{*}$. Since $\sigma_{*}$ measures the bulge and $v_{m}$ reflects the circular velocity of the halo, it is not immediately apparent how the two should be related. This issue was recently addressed by Ho (2007), who showed that although $v_{m}$ does not correlated as tightly with $\sigma_{*}$ as had been thought previously, nevertheless galaxies over a wide range of Hubble types do follow a $v_{m}-\sigma_{*}$ relation. This last step then completes the recipe to transform CO line widths to $\sigma_{*}$.

With these tools in hand, we revisited the CO-based $M_{\bullet}-\sigma_{*}$ relation for quasars. Low-redshift $(z \lesssim 0.2)$ quasars fall roughly on the $M_{\bullet}-\sigma_{*}$ relation of inactive galaxies, albeit with considerable scatter, but high-redshift $(z=1.41-6.42)$ quasars statistically lie displaced from the local relation. Our finding is in qualitative agreement with the results of Shields et al. (2006), but the magnitude of the offset has been reduced with our more refined $\sigma_{*}$ estimates. At a given $\sigma_{*}$, high- $z$ quasars have BH masses larger by a factor of $\sim 4$ relative to local galaxies. Although we cannot rigorously rule out the possibility that we are being misled by a population of quasars with biased orientations, we are encouraged by the fact that our result quantitatively agrees well with that based on the photometric analysis presented by Peng et al. (2006b). The collective evidence suggests that early in the life-cycle of galaxies the development of the bulge lags behind the growth of the central $\mathrm{BH}$.

This work was supported by the Carnegie Institution of Washington and by NASA grants from the Space Telescope Science Institute (operated by AURA, Inc., under NASA contract NAS5-26555). Extensive use was made of the NASA/IPAC Extragalactic Database (NED), which is operated by the Jet Propulsion Laboratory, California Institute of Technology, under contract with NASA. I thank Jenny Greene, Chien Peng, and Greg Shields for discussions and comments on this paper. An anonymous referee provided very helpful suggestions.

\section{REFERENCES}

Aalto, S., Booth, R. S., Black, J. H., \& Johansson, L. E. B. 1995, A\&A, 300, 369

Barnes, J. E., \& Hernquist, L. E. 1996, ApJ, 471, 115

Barth, A. J., Greene, J. E., \& Ho, L. C. 2005, ApJ, 619, L151

Barth, A. J., Martini, P., Nelson, C. H., \& Ho, L. C. 2003, ApJ, 594, L95

Baskin, A., \& Laor, A. 2005, MNRAS, 356, 1029

Bertoldi, F., et al. 2003, A\&A, 409, L47

Bessell, M. S. 2005, ARA\&A, 43, 293

Boroson, T. A., \& Green, R. F. 1992, ApJS, 80, 109

Bushouse, H. A., \& Stanford, S. A. 1992, ApJS, 79, 213

Bushouse, H. A., \& Werner, M. W. 1990, ApJ, 359, 72

Carilli, C. L., \& Wang, R. 2006, AJ, 131, 2763 (erratum: 132, 2231)

Casoli, F., \& Loinard, L. 2001, in Science with the Atacama Large Millimeter Array, ed. A. Wootten (San Francisco: ASP), 305

Courteau, S., McDonald, M., Widrow, L. M., \& Holtzman, J. 2007, ApJ, 655, L21

Croton, D. J. 2006, MNRAS, 369, 1808

de Vaucouleurs, G., de Vaucouleurs, A., Corwin, H. G., Jr., Buta, R. J., Paturel, G., \& Fouqué, R. 1991, Third Reference Catalogue of Bright Galaxies (New York: Springer)

Dickey, J. M., \& Kazés, I. 1992, ApJ, 393, 530

Downes, D., \& Solomon, P. M. 1998, ApJ, 507, 615

Evans, A. S., Frayer, D. T., Surace, J. A., \& Sanders, D. B. 2001, AJ, 121, 1893

Evans, A. S., Solomon, P. M., Tacconi, L. J., Vavilkin, T., \& Downes, D. 2006, AJ, 132, 2398

Ferrarese, L. 2002, ApJ, 578, 90

Ferrarese, L., \& Merritt, D. 2000, ApJ, 539, L9

Ferrarese, L., Pogge, R. W., Peterson, B. M., Merritt, D., Wandel, A., \& Joseph, C. L. 2001, ApJ, 555, L79

Fontanot, F., Monaco, P., Cristiani, S., \& Tozzi, P. 2006, MNRAS, 373, 1173

Gebhardt, K., et al. 2000a, ApJ, 539, L13

. 2000b, ApJ, 543, L5

Glenn, J., \& Hunter, T. R. 2001, ApJS, 135, 177

Greene, J. E., \& Ho, L. C. 2006, ApJ, 641, L2

Greve, T. R., et al. 2005, MNRAS, 359, 1165

Ho, L. C. 2004, ed., Carnegie Observatories Astrophysics Series, Vol. 1: Coevolution of Black Holes and Galaxies (Cambridge: Cambridge Univ. Press)

- 2005, ApJ, 629, 680

- 2007, ApJ, in press (astro-ph/0706.4347)
Ho, L. C., Darling, J., \& Greene, J. E. 2007, ApJ, submitted

Ho, L. C., \& Keto, E. 2007, ApJ, 658, 314

Hopkins, P. F., Hernquist, L., Cox, T. J., Di Matteo, T., Martini, P., Robertson, B., \& Springel, V. 2006, ApJS, 163, 1

Hopkins, P. F., Hernquist, L., Cox, T. J., Robertson, B., \& Krause, E. 2007, ApJ, submitted (astro-ph/0701351)

Kim, D.-C., Vellleux, S., \& Sanders, D. B. 2002, ApJS, 143, 277

Kormendy, J., \& Richstone, D. O. 1995, ARA\&A, 33, 581

Krips, M., Neri, R., Eckart, A., Martín-Pintado, J., Planesas, P., \& Colina, L. 2004, in The Dense Interstellar Medium in Galaxies, ed. S. Pfalzner et al. (Berlin: Springer), 23

Lavezzi, T. E., \& Dickey, J. M. 1997, AJ, 114, 2437

$\longrightarrow$. 1998, AJ, 116, 2672

Magorrian, J., et al. 1998, AJ, 115, 2285

McLeod, K. K., \& Rieke, G. H. 1994, ApJ, 420, 58

- 1995, ApJ, 441, 96

Mouhcine, M., \& Lançon, A. 2003, A\&A, 402, 425

Neugebauer, G., Green, R. F., Matthews, K., Schmidt, M., Soifer, B. T., \& Bennett, J. 1987, ApJS, 63, 615

Paturel, G., Fang, Y., Petit, C., Garnier, R., \& Rousseau, J. 2000, A\&AS, 146, 19

Peng, C. Y., Impey, C. D., Ho, L. C., Barton, E. J., \& Rix, H.-W. 2006a, ApJ, 640,114

Peng, C. Y., Impey, C. D., Rix, H.-W., Kochaneck, C. S., Keeton, C. R., Falco, E. E., Lehár, J., \& McLeod, B. A. 2006b, ApJ, 649, 616

Pizzella, A., Corsini, E., Dalla Bontá, E., Sarzi, M., Coccato, L., \& Bertola, F. 2005, ApJ, 631, 785

Planesas, P., Martín-Pintado, J., Neri, R., \& Colina, L. 1999, Science, 286, 2493

Riechers, D. A., et al. 2006, ApJ, 650, 604

Robertson, B., Hernquist, L., Cox, T. J., Di Matteo, T., Hopkins, P. F., Martini, P., \& Springel, V. 2006, ApJ, 641, 90

Salviander, S., Shields, G. A., Gebhardt, K., \& Bonning, E. W. 2007, ApJ, 662, 131

Sanders, D. B., \& Mirabel, I. F. 1996, ARA\&A, 34, 749

Sanders, D. B., Scoville, N. Z., \& Soifer, B. T. 1991, ApJ, 370, 158

Scharwächter, J., Eckart, A., Pfalzner, S., Moultaka, J., Straubmeier, C., \& Staguhn, J. G. 2003, A\&A, 405, 959

Schöniger, F., \& Sofue, Y. 1994, A\&A, 283, 21

. 1997, A\&A, 323, 14 
Scoville, N. Z., Frayer, D. T., Schinnerer, E., \& Christopher, M. 2003, ApJ, 585, L105

Shields, G. A., Gebhardt, K., Salviander, S., Wills, B. J., Xie, B., Brotherton, M. S., Yuan, J., \& Dietrich, M. 2003, ApJ, 583, 124

Shields, G. A., Menezes, K. L., Massart, C. A., \& Vanden Bout, P. 2006, ApJ, 641,683

Skrutskie, M. F., et al. 2006, AJ, 131, 1163

Sofue, Y. 1992, PASJ, 44, L231

Sofue, Y., \& Rubin, V. C. 2001, ARA\&A, 39, 137

Sofue, Y., Schöniger, F., Honma, M., Tutui, Y., Ichikawa, T., Wakamatsu, K., Kazés, I., \& Dickey, J. 1996, PASJ, 48, 657

Solomon, P. M., Downes, D., Radford, S. J. E., \& Barrett, J. W. 1997, ApJ, 478, 144

Solomon, P. M., \& Vanden Bout, P. 2005, ARA\&A, 43, 677

Treu, T., Malkan, M. A., \& Blandford, R. D. 2004, ApJ, 615, L97
Treu, T., Woo, J.-H., Malkan, M. A., \& Blandford, R. D. 2007, ApJ, in press (astro-ph/0706.0519)

Tremaine, S., et al. 2002, ApJ, 574, 740

Tully, R. B., \& Fisher, J. R. 1977, A\&A, 54, 66

Tutui, Y., \& Sofue, Y. 1999, A\&A, 351, 467

Tutui, Y., Sofue, Y., Honma, M., Ichikawa, T., \& Wakamatsu, K. 2001, PASJ, 53, 701

Verheijen, M. A. W. 2001, ApJ, 563, 694

Walter, F., et al. 2003, Nature, 424, 406

Walter, F., Carilli, C., Bertoldi, F., Menten, K., Cox, P., Lo, K. Y., Fan, X., \& Strauss, M. A. 2004, ApJ, 615, L17

Weiß, A., Downes, D., Neri, R., Walter, F., Henkel, C., Wilner, D. J., Wagg, J., \& Wiklind, T. 2007, A\&A, 467, 955

Weiß, A., Henkel, C., Downes, D., \& Walter, F. 2003, A\&A, 409, L41

Woo, J.-H., Treu, T., Malkan, M. A., \& Blandford, R. D. 2006, ApJ, 645, 900

Wu, X.-B. 2007, ApJ, 657, 177 